\title{
Orientation-contingent color aftereffects are determined by real color, not induced color
}

\author{
W. R. WEBSTER, R. H. DAY, and K. WILLENBERG \\ Monash University, Clayton, Victoria, Australia
}

\begin{abstract}
Three experiments were concerned with whether orientation-contingent color aftereffects (McCollough effects) occur when the perceived colors of differently oriented gratings derive from colored shadows, a compelling instance of simultaneous color contrast. Whereas strong orientationcontingent aftereffects occurred with pink light reflected from vertical gratings viewed in alternation with green light reflected from horizontal gratings, none occurred when pink and green colored shadows were presented with the same physically achromatic gratings (Experiment 1). No aftereffects were evident even after an extended (15-min) period of alternating presentation (Experiment 2). In Experiment 3, colored bars matched in chromaticity to the colored shadows produced strong aftereffects. These outcomes agree with those of Thompson and Latchford (1986), who showed that the McCollough effect is a function of the real color of reflected light, rather than of the apparently constant color of gratings in different illuminants. The implications of the present findings for the neural basis of simultaneous color contrast and the McCollough effect, and for Thompson and Latchford's explanation of the latter, are considered.
\end{abstract}

Perhaps the most striking version of induced color contrast is that produced by the "colored-shadow" method (see Graham \& Brown, 1965; Helmholtz, 1909/1962). An arrangement for producing colored shadows is illustrated in Figure 1. The induced colors are so striking that many observers have difficulty in accepting that the apparently colored regions are physically achromatic. That they are so can be demonstrated by observing the region through a black-lined tube that occludes the colored surround (Helmholtz, 1909/1962). Another form of apparent color has been attributed to color constancy. If a red region is embedded in a Mondrian display (Land, 1983; Zeki, 1980a), it continues to appear red even when it reflects more middle- and than long-wavelength light. Thompson and Latchford (1986) embedded McColloughinducing patterns (red and black vertical gratings and green and black horizontal gratings) in a Mondrian display and found that the orientation-contingent aftereffects (McCollough effects) generated by these patterns were determined by the wavelength of the reflected light, rather than by the apparently constant color of the gratings.

In the case of colored shadows, only white (achromatic) light is reflected from the region covered in shadow. The purpose of the three experiments reported here was to extend the findings of Thompson and Latchford (1986) by ascertaining whether or not orientation-contingent color aftereffects occur when the adaptation stimuli consist of differently oriented grating patterns reflecting only white light but appearing colored. The effects produced by gratings on which induced colors were superimposed were compared with those produced by the same gratings in light of different wavelengths that appeared the same as

Address correspondence to W. R. Webster, Department of Psychology, Monash University, Clayton, Victoria 3168, Australia. the induced colors. For convenience, we will refer to perceived color resulting from colored shadows as apparent color and that from reflected chromatic light as real color.

\section{EXPERIMENT 1}

\section{Method}

Subjects. The 8 volunteer subjects were graduate students and faculty members. All had normal color vision according to the Ishihara plates (Kanehara Shuppan Co., 1974).

Apparatus and Stimuli. The apparatus consisted of two separate units, one designed to generate orientation-contingent color aftereffects and the other to measure their strength by a cancellation procedure.

Apparent colors were induced in the white bars of two $9.5 \times 9.5 \mathrm{~cm}$ achromatic square-wave grating ( 2.18 cycles per degree [cpd]) patterns located $7 \mathrm{~cm}$ apart on a white screen. The subject was seated $1 \mathrm{~m}$ from the screen. The luminance of the apparent colors on the white bars, as measured by a light meter (Minolta Auto-spot II), was $2 \mathrm{~cd} / \mathrm{m}^{2}$. The apparent colors were induced by means of the arrangement illustrated in Figure 1. White light from a lamp illuminated the screen and grating patterns continuously. Then either red light with a dominant wavelength of $620 \mathrm{~nm}$ or green light with a dominant wavelength of $515 \mathrm{~nm}$ (measured by a Minolta Chromameter, Model CL100) was generated by a colored lamp on the floor (the red lamp was on the subject's right and the green lamp was on the subject's left). The lamps could be turned on and off in alternation under computer (DEC Model LSI 11/73) control. The light from the lamps was interrupted by a black opaque square $(10 \times 10 \mathrm{~cm})$, so that a shadow was cast over the appropriate grating while the surrounding screen was illuminated by red or green light mixed with white (see Figure 1). The patterns, therefore, remained achromatic but appeared colored by induction from the surround. With the red lamp on, the white bars of the horizontal grating on the left appeared to be pale green; when the green lamp was on, the white bars of the vertical grating on the right appeared to be pink. To produce real colors with the same gratings, the white light was turned off, so that the computer-controlled red or green 


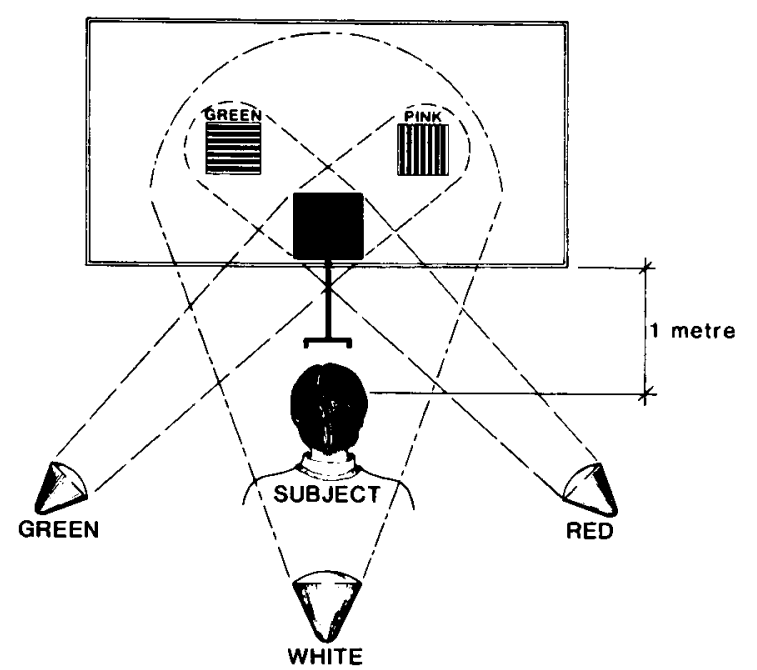

Figure 1. Schematic diagram of experimental arrangement for generating colored shadows and real colors on vertical and horizontal gratings. For details see text. When the green and white lights are operational, the screen is illuminated by pale green light. The region in the shadow of the shadow caster appears pink by contrast; this is the apparent color, or colored shadow effect. Likewise, when the red and white lights are on, the screen is illuminated by pink light and the region in shadow appears pale green by contrast. The same regions were pink or green from reflected light when the red light and green lights, respectively, were directed onto the gratings in the absence of the influence of the shadow caster.

lamp directly illuminated the vertical or horizontal grating together with the surrounding screen. The luminance of the vertical redilluminated grating was about $6.6 \mathrm{~cd} / \mathrm{m}^{2}$, and that of the horizontal green-illuminated grating was about $5.9 \mathrm{~cd} / \mathrm{m}^{2}$.

The apparatus used to measure the strength of the orientationcontingent aftereffect has already been described (Webster, Crassini, \& Willenberg, 1987). Briefly, a red-green-blue (RGB) monitor (Barco, Model GD 33) was linked to a computer interfaced with a real-time image-processing system (Image Technology Inc., Model AP-512). A vertical and a horizontal grating of the same spatial frequency as those on the screen were displayed side by side on the monitor. The subjects could alter the color of either grating by pressing one of three colored buttons (red, green, and white). The red and green buttons controlled the combined output of the red, green, and blue guns of the monitor. The subject's task throughout was to cancel the apparent color of the gratings until the colored bars appeared to be white. If the bars appeared red, they could be made to appear progressively less saturated to the point of whiteness by pressing the red button. When the point of whiteness was reached, the subject pressed the white button. If the subject went too far (i.e., "overshot" so that the bars appeared to be green), he/she pressed the green button until the bars appeared again to be white. The degree of change required to achieve apparent whiteness of the apparently colored bars was recorded and stored by the computer. The output of the three guns of the monitor was yoked by software so that the relationship between output and buttonpresses was always linear (see below). The change in color appearance associated with each buttonpress was below threshold, thus ensuring that subjects could not use a strategy based on counting the number of buttonpresses.

Calibrations. The chromaticity of the monitor screen as a function of voltage change to the guns was measured by the chromameter. The resulting. CIE coordinates are set out in Figure 2. Although measures were taken after each buttonpress, in the in- terest of clarity only every 10 th measure is plotted in Figure 2. Pressing the red button increased the proportion of green in the stimulus, thus canceling the red color perceived by the subject. The changes are nonlinear in the direction of green over the full range of buttonpresses. However, over a range of 40 presses, each press clearly produced a linear change in CIE coordinates. This range is more than double the maximum number of buttonpresses required at any time to cancel red to white. A least squares curve-fitting program showed a strong linear trend ( $y=1.35-3.98 x$; coefficient of determination $=.99$ ). A similar relationship resulted from pressing the green button to cancel the green in linear steps toward red. A least squares fit for $\mathbf{4 0}$ presses also yielded a linear trend ( $y=$ $0.72-1.61 x$; coefficient of determination $=1.00$ ). Both linear equations are plotted in Figure 2. Thus a red color was canceled in linear steps toward greenness and a green color was canceled in linear steps toward redness. Given these linear relationships, the number of buttonpresses as a relative measure of apparent color change is justified.

Five subjects were required to match on the monitor the color of both the apparent and the real colors projected on the screen. It was not possible to seat the subjects at the same distance from both the screen and the monitor. They were first seated $2 \mathrm{~m}$ from the screen and $1 \mathrm{~m}$ from the monitor, and were asked to make color judgments. They then made another set of judgments at both $1 \mathrm{~m}$ and $2 \mathrm{~m}$ from the screen. These judgments revealed no change in either real or apparent color with distance. The computer values for the matchings were transformed to CIE coordinates using the linear relationships established above. For the apparent colors, red had average CIE coordinates of $x=.293, y=.251$, and green had average coordinates of $x=.260, y=.318$. For real colors, red had average coordinates of $x=.341, y=.214$, and green had average coordinates of $x=.257, y=.389$. Thus the real colors were matched as considerably redder and greener than the apparent colors.

Procedure. The procedure involved two stages: induction and measurement of an orientation-contingent aftereffect with apparent colors, followed by induction and measurement of an orientationcontingent aftereffect with real colors. Testing was always in that order. In both induction series, each subject saw red-and-black vertical gratings and green-and-black horizontal gratings, in that order. Both stages consisted of a pretest, induction, a 1-min rest (to allow time for the dissipation of afterimages), and a posttest. For the pretest, the subject was seated $1 \mathrm{~m}$ from the monitor, on which both gratings were displayed side by side, vertical to the right, horizontal to the left. If either grating designated by the experimenter appeared white, the subject pressed one of the colored buttons at random, and then the white button. If either grating appeared colored, the subject canceled the color in the manner described above.

For the induction phase, the subject moved to a seated position $1 \mathrm{~m}$ from the white screen. The subject was instructed to alternate the direction of his/her gaze from one grating to the other as each became colored. For the apparent colors, the white bars of the vertical grating on the right were made pink by induction as described above, and those of the horizontal grating on the left were made pale green for 1010 -sec alternating periods. Thus the total induction period was $3 \mathrm{~min} 20 \mathrm{sec}$. At the end of the induction period the subject sat in white light for $1 \mathrm{~min}$ to allow any negative afterimages to dissipate. A similar procedure was followed when the vertical grating was illuminated by red light and the horizontal grating by green light.

The posttest was the same as the pretest. The subject moved again to his/her position in front of the monitor and followed the procedure described above for canceling the color of the gratings until they appeared to be white.

Measurements. The pretest score was subtracted from the posttest score for each subject. A resultant positive score indicated that during the posttest the grating had appeared to be green and there- 


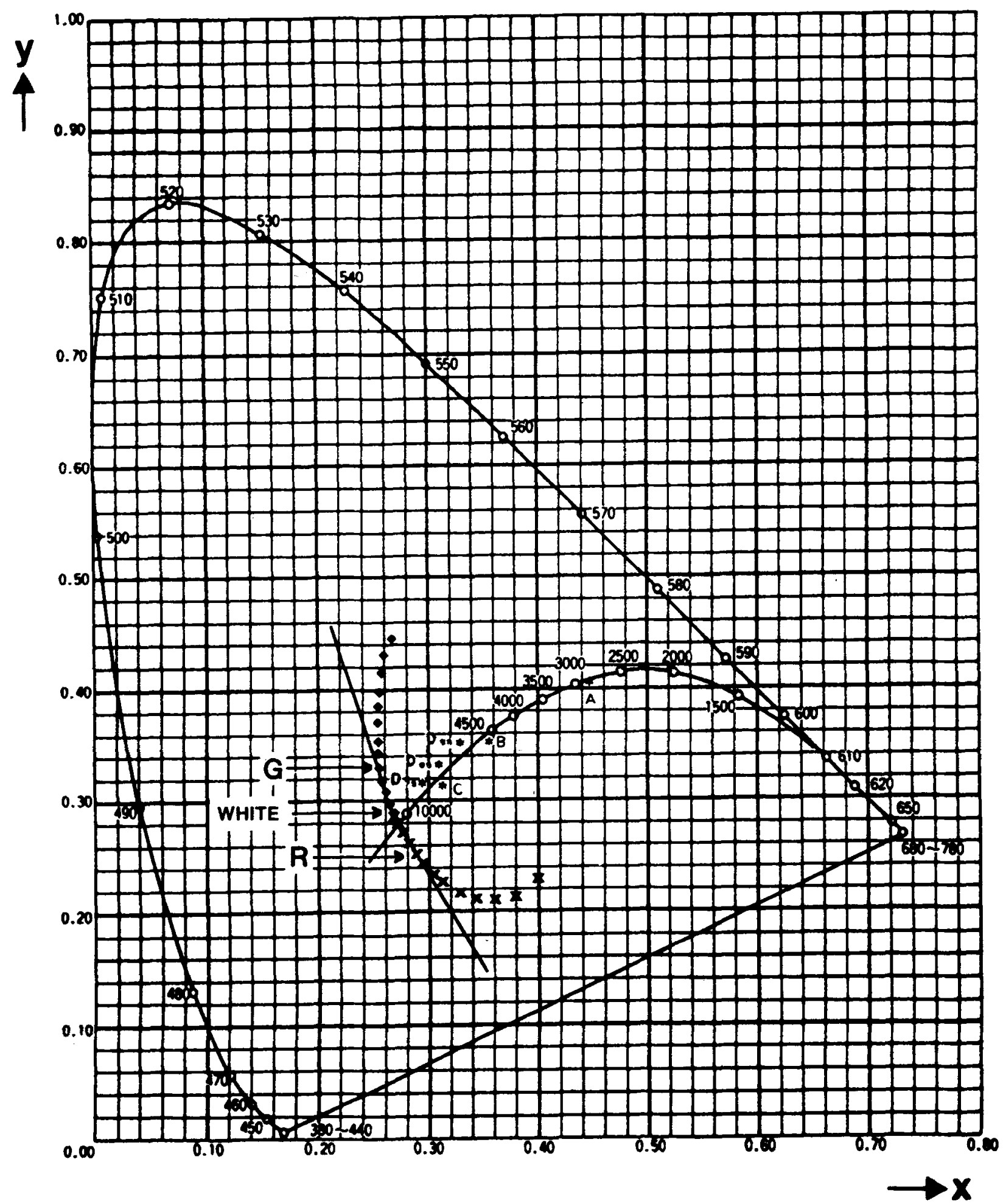

Figure 2. Plots of CIE coordinates of the monitor screens obtained from a chromameter after each buttonpress on the color cancellation system. The coordinates are plotted directly on a two-dimensional chromaticity CIE diagram. The diamond points indicate the coordinates of every 10th press of the red button, which canceled any apparent red color by increasing the proportion of green. The crosses indicate the coordinates of every 10 th press of the green button, which canceled any apparent green color by increasing the proportion of red. The straight lines are fitted by the linear equations provided in the text. The arrow marked WHITE indicates the coordinate position of white on the monitor screen. Arrows marked $G$ and $R$ indicate the positions achieved after 40 buttonpresses, and show the range over which the linear equations were calculated. 
fore the subject had added more red to cancel to apparent white. A negative score indicated that the grating had appeared to be red and therefore the subject had added more green to cancel to apparent white. The positive and negative signs were retained in the statistical analysis.

\section{Results and Discussion}

The results are shown in Figure 3. Orientationcontingent aftereffects are clearly evident for induction with real colors, but not with apparent colors. In the former condition the mean score of $\mathbf{+ 5 . 8}$ for the vertical grating indicates that in the posttest the grating appeared green and therefore required the addition of red to cancel to white. Likewise, the mean score of -6.13 for the horizontal grating indicates that in the posttest it appeared red and therefore required the addition of green to cancel to white. In the apparent color condition the mean score was +.12 for the vertical grating and -.12 for the horizontal grating. Since our interest centered primarily on whether aftereffects occur with apparent colors, we tested the difference of each of the mean scores from zero by means of $t$ tests for correlated scores. For real colors the difference proved to be significant for both the vertical (V) and the horizontal (H) gratings $\left[t_{\mathrm{v}}(7)=4.86\right.$, $p<.01 ; t_{\mathrm{H}}(7)=3.35, p<.01$ ]. For apparent colors both differences fell well short of significance $\left[t_{\mathrm{v}}(7)=\right.$ $\left..22, p>.05 ; t_{\mathrm{H}}(7)=.15, p>.05\right]$.

The apparent colors generated by the colored-shadow technique were perceptually compelling. Nevertheless, the data are quite clear in showing that the apparent colors, unlike real colors, combined with vertical and horizontal gratings and presented in an alternating fashion do not induce McCollough-type aftereffects. Real colors under the same conditions clearly did so. However, it can be seen in Figure 3 that although the means for apparent colors were not significantly different, they were in the same direction as those for real colors. This outcome raised the possibility that more protracted induction with apparent colors might give rise to significant effects. This possibility was tested in the second experiment.

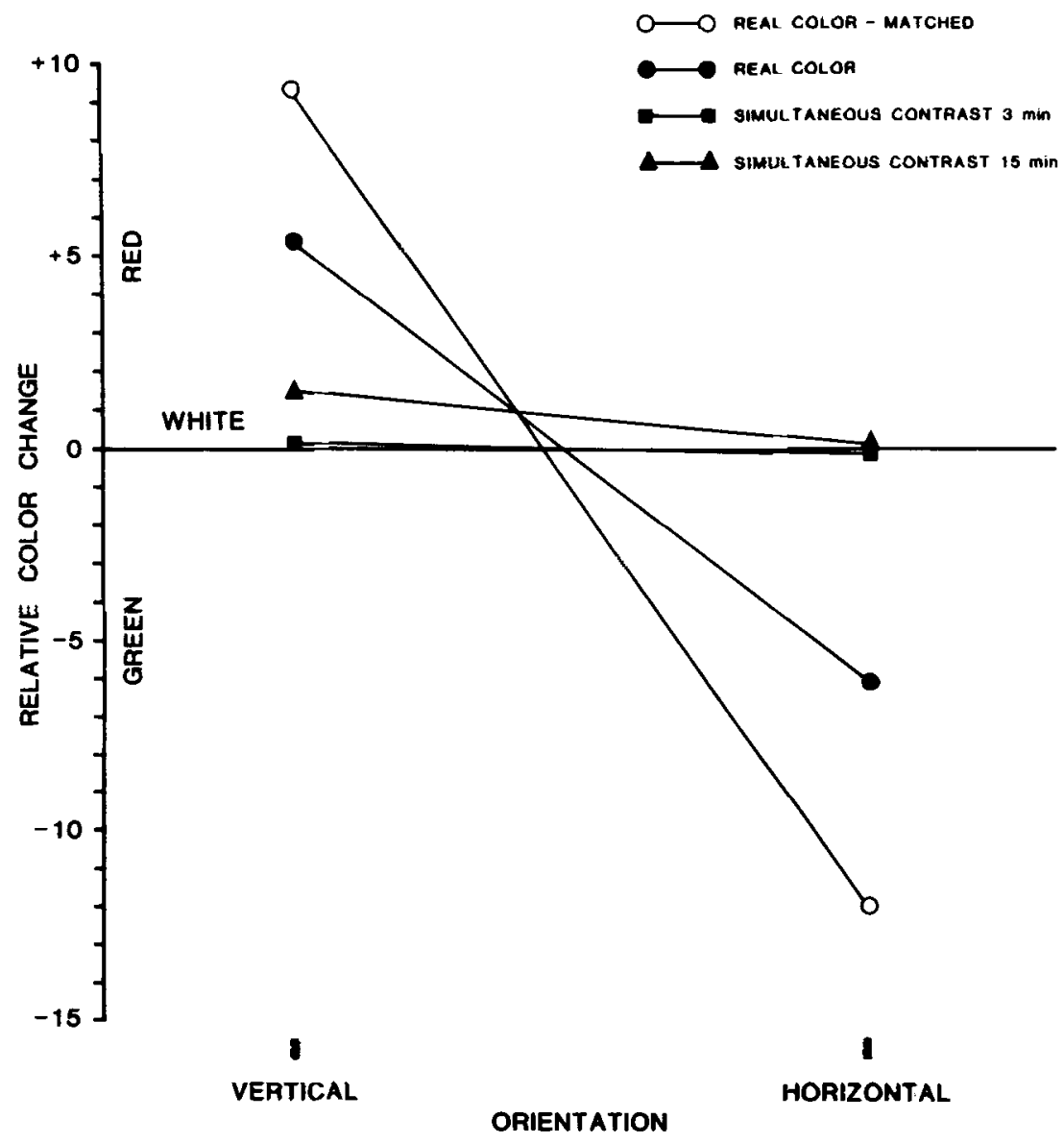

Figure 3. Means of linear changes in color needed to make an achromatic test grating appear white after adaptation. A pasitive score indicates that the subject saw green, and more red was required to cancel to white. A negative score indicates that the subject saw red, and more green was required to cancel to white. $\bullet$, Exposure to real colors for $3 \mathrm{~min}$; $\bullet$, exposure to apparent colors for $3 \mathrm{~min}$; $\Delta$, exposure to apparent colors due to colored shadows for $15 \mathrm{~min} ; \circ$, exposure to matched colors for $15 \mathrm{~min}$. 


\section{EXPERIMENT 2}

The aim of Experiment 2 was to establish whether significant orientation-contingent aftereffects with apparent colors generated by the colored-shadow technique would occur after a 15-min induction phase. The apparatus and procedure were the same in all respects as in Experiment 1 . There was only one induction stage, that with apparent colors, generated in the same manner as in Experiment 1. Repetition of induction with real colors was not considered necessary in view of the strong effect obtained in Experiment 1. The vertical and horizontal gratings were each presented 45 times, resulting in a total induction period of $15 \mathrm{~min}$. There were 6 subjects, all faculty members who were not familiar with the McCollough effect. According to the Ishihara test, the color vision of all subjects was normal.

The results are shown in Figure 3. The mean score was +1.50 for the vertical grating and 0 for the horizontal grating. Neither score was significantly different from zero. We can therefore confidently conclude that orientationcontingent aftereffects of color do not occur, even after a protracted induction period, with apparent colors in the form of colored shadows.

\section{EXPERIMENT 3}

In Experiment 1 the calibration of the apparent colors induced by the colored-shadow technique and that of the real colors revealed a considerable difference in chromaticity. The real colors gave rise to a McCollough effect in Experiment 1, whereas the apparent colors did not. It was possible, therefore, that the presence and absence of a McCollough effect under conditions of real-color and apparent-color induction, respectively, were due to differences in chromaticity. The aim of Experiment 3 was to test this possibility. Real colors of the same saturation as the apparent colors were used. Apparent and real colors were matched on the monitor screen and the resulting values of the former were used as adapting stimuli.

\section{Method}

Six subjects, graduate students and faculty with normal color vision, matched the apparent colors of the colored shadows on the monitor. As with the calibration procedures, each subject sat $1 \mathrm{~m}$ from the monitor and $2 \mathrm{~m}$ from the screen on which the colored shadows were generated. Therefore, in retinal terms the gratings were $2.18 \mathrm{cpd}$ for the monitor and double that frequency for the screen. The subjects judged the color of the apparent colors at 1 and $2 \mathrm{~m}$. All subjects reported that there was no change of hue as a function of distance. The subjects then viewed a black and white vertical grating on the monitor and pressed the red button until the grating's real color matched the apparent red color on the screen; then they matched a black and white horizontal grating to the apparent green color by pressing the green button. The values of the individual matches were stored in the computer and later used to generate the colored adapting stimuli individually for each subject. The subjects were again seated $1 \mathrm{~m}$ from the monitor and pretest stimuli were presented as before. Judgments of color were then obtained. The matched red and black vertical grating and the matched green and black horizontal grating were then presented in alternation for periods of $10 \mathrm{sec}$ each. A series of 45 alternations of these gratings resulted in a 15 -min induction period. After a 1-min interval, posttest judgments were obtained. The cancellation and scoring procedures were the same as those used in the earlier experiments.

\section{Results and Discussion}

The vertical test gratings appeared green (mean score $=-12.0$ ) and the horizontal test gratings appeared red (mean score $=9.33$ ). Both were significantly different from zero $\left[t_{\mathrm{V}}(5)=2.71, p<.05 ; t_{\mathrm{H}}(5)=3.16\right.$, $p<.05$ ]. This shows that when the real colors were matched in chromaticity to the apparent colors they continued to give rise to McCollough effects. It can therefore be concluded that the results from Experiments 1 and 2 were not a function of the differences in chromaticity between the real and apparent colors of the grating patterns.

\section{GENERAL DISCUSSION}

In Experiments 1 and 2, the subjects reported that apparent colors-that is, the so-called colored shadows-in physically achromatic regions of the stimulus display were vivid and strong. Even so, the data are quite clear in showing that when combined with vertical and horizontal gratings, pink and green colored shadows do not produce orientation-contingent aftereffects of the kind first demonstrated by McCollough (1965). The results also show that real colors from pink and green light reflected from the gratings, when presented under the same conditions, produce strong McCollough effects. The results of Experiment 2 indicate that even after a 15-min induction period orientation-contingent color aftereffects do not occur with apparent colors. The results of Experiment 3 are also clear-cut. Real colors, carefully matched in chromaticity to the apparent colors, produced strong McCollough effects. That is, no aftereffects were induced by the apparent colors in Experiment 1, whereas strong aftereffects were induced by their chromatic equivalents in real color. This result rules out any possibility that the failure of colored shadows to produce an aftereffect might be a function of their relatively low saturations. The matched colors produced a larger aftereffect than the more saturated real colors used in Experiment 1. This difference is presumably due to the longer exposure duration used with the matched colors (Skowbo \& White, 1983).

The outcomes of these experiments accord well with those of Thompson and Latchford (1986). In their experiment, a red and black vertical grating and a green and black horizontal grating were embedded in a Mondrian display (Land, 1983). The whole array was illuminated with predominantly red or green light; however, the gratings continued to appear red or green even though they reflected more middle- than long-wavelength light or more long- than middle-wavelength light, respectively. After the usual alternating induction procedure, the McCollough effect was in accord with expectations from the color of 
the reflected light (i.e., real color), not with those from apparent color. The main difference between Thompson and Latchford's (1986) experiment and those reported here is that in the former, the red and black and green and black gratings looked red and black while reflecting mainly green light, or green and black while reflecting mainly red light. In the experiments described here, black and white gratings looked pink or green while reflecting white light. In Thompson and Latchford's experiment, McCollough effects occurred in accord with the color of the reflected light; in the experiments reported here, McCollough effects did not occur with white light, which, of course, is to be expected.

Even though Thompson and Latchford (1986) acknowledged that cells in cortical area V1 respond to reflected light, they preferred to interpret their results in terms of the presence of cells responsive to apparent color in cortical area V4. Zeki (1980a) described V4 cells that respond with constant output to a Mondrian display under light of different wavelengths. Thompson and Latchford suggested that if, for example, a red vertical grating is placed in green light, observers behave as if they learn to compensate for the excess of green light when they view the test grating. According to this suggestion, the McCollough effect can be regarded as a form of orientationcontingent color constancy having its basis in the cells described by Zeki (1980a) in V4. (Note that Zeki's claim that V4 cells are very color sensitive has been strongly challenged by Schein, Marrocco, and de Monasterio, 1982.)

Colored shadows have traditionally been regarded as instances of simultaneous contrast; nevertheless, a relationship between color contrast and color constancy has long been recognized (Graham \& Brown, 1965; Hochberg, 1971; Richards, 1977). The question therefore arises as to whether the results of the three experiments reported here can be interpreted in terms of cells responsive to apparent color in area V4, as proposed by Thompson and Latchford (1986). On first consideration, the evidence in favor of this view is encouraging. Colored shadows extend over considerable areas of the visual field, making them a powerful demonstration of the "filling-in" process described by Walls (1954) and Gerrits and Vendrik (1970). Informal observations during the course of the three experiments showed that the effect can extend to areas subtending up to at least $23^{\circ}$ square. Indeed, the limit seemed to be set only by the area of colored shadows that could be generated. Many V4 cells have receptive fields that are up to 36 times the area of those in V1 (Desimone, Schein, Moran, \& Ungerleider, 1985). Furthermore, Zeki (1980b) claimed that there are cells in V4 with relatively large receptive fields $\left(4 \times 3.5^{\circ}\right)$ that are responsive to the apparent colors of colored shadows.

Both Richards (1977) and Gouras (1974) argued that the opponent-process receptive fields in the primate retina and lateral geniculate could not be the basis of simultaneous color contrast. Richards proposed that to mediate simultaneous color contrast, receptive fields must have the following characteristics: (1) a filtering operation that discounts both the intensity and the spectral composition of ambient illumination; (2) an ability to reduce the role of borders in contrast, since contrast is strongest when luminance steps are minimized; (3) an opponent-process character discounting ambient color and enhancing any color differences. These specifications are met by concentric double-opponent cells with, for example, both red "on" and green "off" centers and both red "off" and green "on" surrounds described by Hubel and Wiesel (1968) and Michael (1978a) in area V1. However, these cells lack the orientation sensitivity required to mediate the McCollough effect.

Michael (1978b) reported some preliminary evidence that the double-opponent simple cells in V1 could be mediating the McCollough effect. Although these cells combine the necessary sensitivity to orientation with the appropriate opponent-process features for contrast, they lack one important characteristic of colored shadows. The size of their receptive fields $\left(3^{\circ}-10^{\circ}\right)$ is too small to account for the size of colored shadows. Unfortunately, recent work on V4 cells (Schein et al., 1982) has also shown that their receptive fields lack the necessary sensitivity to orientation and opponent processes, even when the receptive fields are of an appropriate size. It seems that neither VI simple cells nor V4 cells have all the necessary properties to mediate both the McCollough effect and the simultaneous color contrast. This renders Thompson and Latchford's (1986) hypothesis involving V4 cells difficult to sustain.

It should not be concluded from Thompson and Latchford's (1986) results or from those reported here that apparent color in any form cannot give rise to orientationcontingent aftereffects. In a much neglected study, Murch and Hirsch (1972) used the apparent color of a negative afterimage to induce McCollough effects. A red afterimage was superimposed on vertical bars, and a green afterimage on horizontal bars. Following induction, vertical test gratings were seen as green and horizontal test gratings as pink. It seems, therefore, that whereas simultaneous contrast as manifested in colored shadows does not produce orientation-contingent color aftereffects, successive contrast in the form of afterimages does. (Incidentally, because of the implications of Murch and Hirsch's finding for the work described here, we repeated their experiment in our laboratory, using the method for measuring the contingent aftereffect described above. The original results were confirmed.)

In Experiments 1 and 2 described here, both adaptation gratings were visible simultaneously, and the subjects were required to move their gaze from one to the other as they were made colored under computer control. It was stressed that the subjects should concentrate on each grating in turn, and this appears to have happened, inasmuch as the real colors produced significant aftereffects. It might be argued that because the colored shadows were surrounded by light of a complementary color, eye movements to positions outside the gratings could lead to can- 
cellation of any aftereffect. We feel this is unlikely, inasmuch as there is no evidence that interspersed homogeneous colors cancel the McCollough effect. Even if eye movements did occur, when the gaze returned to the gratings in the shadow (which were actually achromatic), a negative afterimage would have been present and this would have tended to enhance any aftereffect (Murch \& Hirsch, 1972).

It is generally accepted that the neural events mediating the experience of afterimages originate in the retina (Craik, 1940). It therefore seems that neural activity originating in the retina and activity associated with orientation processing at a higher level in the visual system can interact, in a manner as yet unspecified, to generate orientation-contingent effects. Thus, even if simultaneous color contrast were of retinal origin, as some evidence suggests (De Valois \& Walraven, 1967; Poppel, 1986), this fact in itself would not ensure a failure to produce orientation-contingent effects.

\section{REFERENCES}

CRaIK, K. J. W. (1940). Origin of visual afterimages. Nature, 145, 512-513.

Desimone, R., Schein, S. J., Moran, J., \& Ungerleider, L. G. (1985). Contour, color and shape analysis beyond the striate cortex. Vision Research, 25, 441-452.

De Valois, R. L., \& Walraven, J. (1967). Monocular and binocular aftereffects of chromatic adaptation. Science, 155, 463-465.

Gerrits, H. J. M., VendrIK, A. J. H. (1970). Simultaneous contrast: Filling-in process and information processing in man's visual system. Experimental Brain Research, 11, 411-430.

Gouras, P. (1974). Opponent-color cells in different layers of foveal striate cortex. Journal of Physiology, 238, 583-602.

Graham, C. H., \& Brown, J. L. (1965). Color contrast and color appearances: Brightness constancy and color constancy. In C. H. Graham (Ed.), Vision and visual perception (pp. 452-478). New York: Wiley.

' Helmholtz, H. von. (1962). Treatise on physiological optics (3rd ed., J. P .C. Southall, Ed. \& Trans.). New York: Dover. (Original work published 1909)

Hochberg, J. (1971). Perception I. Color and shape. In J. W. Kling
\& L. R. Riggs (Eds.), Woodworth and Schlosberg's experimental psychology (pp. 395-474). London: Methuen.

HubEL, D. H., \& WIESEL, T. N. (1968). Receptive fields and functional architecture of monkey striate cortex. Journal of Physiology, 195, 215-243.

LAND, E. H. (1983). Recent advances in retinex theory and some implications for cortical computations: Color vision and the natural image. Proceedings of the National Academy of Science (USA), 80, $5163-5169$.

McCollough, C. (1965). Color adaptation of edge-detectors in human visual system. Science, 149, 1115-1116.

MiCHAEL, C. R. (1978a). Color vision mechanisms in monkey striate cortex: Dual-opponent cells with concentric receptive fields. Journal of Neurophysiology, 41, 572-588.

MiCHAEL, C. R. (1978b). Color vision mechanisms in monkey striate cortex in simple cells with dual opponent-color receptive fields. Joumal of Neurophysiology, 41, 1233-1249.

MurCH, G. M., \& Hirsch, J. (1972). The McCollough effect created by complementary afterimages. American Joumal of Psychology, 85, 241-247.

POPPEL, E. (1986). Long-range colour-generating interactions across the retina. Nature, 320, 523-525.

RichARDS, W. (1977). Lessons in constancy from neurophysiology. In W. Epstein (Ed.), Stability and constancy in visual perception: Mechanisms and processes (pp. 421-436). New York: Wiley.

Schein, S. J., Marrocco, R. T., \& De Monasterio, F. M. (1982). Is there a high concentration of color-selective cells in area V4 of monkey visual cortex? Journal of Neurophysiology, 47, 193-213.

SKowbo, D., \& WHITE, K. (1983). McCollough effect acquisition depends on duration of exposure to inducing stimuli, not number of stimulus presentations. Perception \& Psychophysics, 34, 549-551.

ThOMPSON, P., \& LATCHFORD, G. (1986). Colour-contingent after-effects are really wavelength contingent. Nature, 320, 525-526.

WALLS, G. L. (1954). The filling-in process. American Joumal of Optometry, 31, 329-341.

Webster, W. R., Crassini, B., Willenberg, K. (1987). Simultaneous color contrast produced by McCollough effects are spatially contingent. Perception \& Psychophysics, 41, 402-408.

ZEKI, S. (1980a). The representation of colours in the cerebral cortex. Nature, 284, 412-418.

ZEKI, S. (1980b). The response of colour-specific cells in monkey visual cortex to colours produced by shadows. Journal of Physiology, 306, 29-30P.

(Manuscript received October 1, 1986; revision accepted for publication November 23, 1987.) 